Research Article

\title{
The Characterizing Properties of (Signless) Laplacian Permanental Polynomials of Almost Complete Graphs
}

\author{
Tingzeng $W u$ iD and Tian Zhou \\ School of Mathematics and Statistics, Qinghai Nationalities University, Xining, Qinghai 810007, China \\ Correspondence should be addressed to Tingzeng Wu; mathtzwu@163.com
}

Received 16 August 2021; Accepted 17 September 2021; Published 30 September 2021

Academic Editor: Barbara Martinucci

Copyright (c) 2021 Tingzeng $\mathrm{Wu}$ and Tian Zhou. This is an open access article distributed under the Creative Commons Attribution License, which permits unrestricted use, distribution, and reproduction in any medium, provided the original work is properly cited.

Let $G$ be a graph with $n$ vertices, and let $L(G)$ and $Q(G)$ denote the Laplacian matrix and signless Laplacian matrix, respectively. The Laplacian (respectively, signless Laplacian) permanental polynomial of $G$ is defined as the permanent of the characteristic matrix of $L(G)$ (respectively, $Q(G)$ ). In this paper, we show that almost complete graphs are determined by their (signless) Laplacian permanental polynomials.

\section{Introduction}

We use $G$ to denote a simple graph with vertex set $V(G)=$ $\left\{v_{1}, v_{2}, \ldots, v_{n}\right\}$ and edge set $E(G)=\left\{e_{1}, e_{2}, \ldots, e_{m}\right\}$. The degree of a vertex $v \in V(G)$ is denoted by $d(v)$. The degree matrix of $G$, denoted by $D(G)$, is the diagonal matrix whose $(i, i)$ th entry is $d\left(v_{i}\right)$. For a subgraph $H$ of $G$, let $G-E(H)$ denote the subgraph obtained from $G$ by deleting the edges of $H$. Let $c_{i}(G)$ and $p_{i}(G)$ denote, respectively, the numbers of $i$-cycles and $i$-vertex paths in $G$. Let $c_{3}\left(G_{v}\right)$ denote the number of triangles containing the vertex $v$ of $G$. Let $G \cup H$ be the union of two graphs $G$ and $H$ which have no common vertices. For any positive integer $l$, let $l G$ be the union of $l$ disjoint copies of graph $G$. For convenience, the complete graph, path, cycle, and star on $n$ vertices are denoted by $K_{n}$, $P_{n}, C_{n}$, and $K_{1, n-1}$, respectively.

The permanent of $n \times n$ matrix $X=\left(x_{i j}\right)$ $(i, j=1,2, \ldots, n)$ is defined as

$$
\operatorname{per}(X)=\sum_{\sigma} \prod_{i=1}^{n} x_{i \sigma(i)},
$$

where the sum is taken over all permutations $\sigma$ of $\{1,2, \ldots, n\}$. Valiant [1] has shown that computing the permanent is \#P-complete even when restricted to $(0,1)$ matrices.
Let $M$ be an $n \times n$ matrix. The permanental polynomial of $M$, denoted by $\pi(M, x)$, is defined to be the permanent of the characteristic matrix of $M$, i.e.,

$$
\pi(M)=\pi(M, x)=\operatorname{per}(x I-M),
$$

where $I$ is the identity matrix of size $n$.

Let $A(G)$ denote the adjacency matrix of $G$. The Laplacian matrix $L(G)$ and signless Laplacian matrix $Q(G)$ of $G$ are defined by $L(G)=D(G)-A(G)$ and $Q(G)=D(G)+$ $A(G)$, respectively. We call $\pi(L(G), x)(\operatorname{resp}, \pi(Q(G), x))$ the Laplacian (resp, signless Laplacian) permanental polynomial of $G$. The Laplacian permanental polynomial of a graph was first considered by Merris et al. [2], and the signless Laplacian permanental polynomial was first studied by Faria [3]. For more studies on (signless) Laplacian permanental polynomials, see [4-14], among others.

Two graphs $G$ and $H$ are called Laplacian copermanental if $\pi(L(G), x)=\pi(L(H), x)$. Analogously, signless Laplacian copermanental could be defined. A graph $G$ is said to be determined by its Laplacian (resp, signless Laplacian) permanental polynomial if any graph Laplacian (resp, signless Laplacian) copermanental with $G$ is isomorphic to $G$.

It is interesting to characterize which graph is determined by graph polynomials [15-18]. Merris et al. [2] first discussed the problem: which graph is determined by its 
Laplacian permanental polynomial? Answer to the problem is very hard. Up to now, only a few results are known about the problem. Merris et al. computed the Laplacian permanental polynomials of all connected graphs on 6 vertices, and they found that there exist no nonisomorphic Laplacian copermanental graphs of such graphs. Based on the result, they stated that they do not know of a pair of nonisomorphic Laplacian copermanental graphs. Recently, Liu [19] showed that complete graph $K_{n}$ and star $S_{n}$ are determined by their (signless) Laplacian permanental polynomials.

Let $\mathscr{G}_{n}$ denote the set of graphs each of which is obtained from $K_{n}$, by removing five or fewer edges. Cámara and Haemers [20] showed that all graphs in $\mathscr{G}_{n}$ are determined by their characteristic polynomials of adjacency matrices of these graphs. The authors [21] proved that all graphs in $\mathscr{G}_{n}$ are determined by their $A_{\alpha}$-spectra. In this paper, our interest is to discuss which graph in $\mathscr{G}_{n}$ is detertmined by its (signless) Laplacian permanental polynomial. And, we prove the following result.

Theorem 1. All graphs in $\mathscr{G}_{n}$ are determined by their (signless) Laplacian permanental polynomial.

The rest of this paper is organized as follows. In Section 2 , we present some characterizing properties of the (signless) Laplacian permanental polynomial and give some structural properties of graphs in $\mathscr{G}_{n}$. In Section 3, we give the Proof of Theorem 1.

\section{Preliminaries}

Let $\mathscr{G}_{n}$ denote the set of graphs each of which is obtained from $K_{n}$ by removing five or fewer edges. For $n \geq 10$, there exist exactly 45 nonisomorphic graphs each of which is obtained from $K_{n}$ by removing five or fewer edges [21, 22]. These graphs are labeled by $G_{i j}, 1 \leq i \leq 5,0 \leq j \leq 25$, and illustrated in Figure 1. For some properties of graphs in $\mathscr{G}_{n}$, see $[21,22]$, among others.

Lemma 1 (see [22]). Let $H \subseteq K_{n}$ be a graph with l edges and let $G=K_{n}-E(H)$. Then,

$$
c_{3}(G)=\left(\begin{array}{c}
n \\
3
\end{array}\right)-l(n-2)+\sum_{v \in V(H)}\left(\begin{array}{c}
d(v) \\
2
\end{array}\right)-c_{3}(H) .
$$

In [22], the first author calculated the number of triangles of some $G \in \mathscr{G}_{n}$, see Table 1 .

Lemma 2 (see [22]). Let $H \subseteq K_{n}$ be a graph with $l$ edges and let $G=K_{n}-E(H)$. Then,

$$
c_{4}(G)=3\left(\begin{array}{l}
n \\
4
\end{array}\right)-2 l\left(\begin{array}{c}
n-2 \\
2
\end{array}\right)+\left[2\left(\begin{array}{l}
l \\
2
\end{array}\right)+(n-5) \sum_{v \in V(H)}\left(\begin{array}{c}
d(v) \\
2
\end{array}\right)\right]-p_{4}(H)+c_{4}(H)
$$

In [22], the first author calculated the number of quadrangles of some $G \in \mathscr{G}_{n}$, see Table 2 .

Lemma 3 (see [21]). Let $c_{3}\left(G_{v}\right)$ denote the number of triangles containing the vertex $v$ of $G$. Using the principle of inclusion-exclusion, we can obtain the following result. Let $H \subseteq K_{n}$ be a graph with $k$ edges and let $G=K_{n}-E(H)$. Let $v \in V(G)$ and let $v$ be an endpoint of $l$ edges in $E(H)$. Then,

$$
c_{3}\left(G_{v}\right)=\left(\begin{array}{c}
n-1 \\
2
\end{array}\right)-(k-l)-l(n-1-l)-\left(\begin{array}{l}
l \\
2
\end{array}\right)+c_{3}\left(\bar{G}\left(v_{i}\right)\right)+\left|P_{3}\right|
$$

Lemma 4 (see [19]). Let $G$ be a graph with $n$ vertices and $m$ edges, and let $\left(d_{1}, d_{2}, \ldots, d_{n}\right)$ be the degree sequence of $G$. Suppose that $\pi(L(G), x)=\sum_{i} p_{i}(G) x^{n-i}$. Then,

(i) $p_{0}(G)=1$

(ii) $p_{1}(G)=-2 m$

(iii) $p_{2}(G)=2 m^{2}+m-(1 / 2) \sum_{i} d_{i}^{2}$

(iv) $\begin{aligned} & p_{3}(G)=-(1 / 2) \sum_{i} d_{i}^{3}+(m+1) \sum_{i} d_{i}^{2}-(4 / 3) m^{3}- \\ & 2 m^{2}+2 c_{3}(G)\end{aligned}$

(v) $p_{4}(G)=-(1 / 4) \sum_{i} d_{i}^{4}+((2 / 3) m+1) \sum_{i} d_{i}^{3}-(1 / 2)$ $\left(2 m^{2}+5 m+1\right) \sum_{i} d_{i}^{2}+(1 / 8)\left(\sum_{i} d_{i}^{2}\right)^{2}+\sum_{\left(v_{i} v_{j}\right) \in E(G)}$ $d_{i} d_{j}+2 \sum_{i} d_{i} c_{3}\left(G_{v_{i}}\right)+2 c_{4}(G)-4 m c_{3}(G)+(2 / 3)$ $m^{4}+2 m^{3}+(1 / 2) m^{2}+(1 / 2) m$
Lemma 5 (see [19]). Let $G$ be a graph with $n$ vertices and $m$ edges, and let $\left(d_{1}, d_{2}, \ldots, d_{n}\right)$ be the degree sequence of $G$. Suppose that $\pi(Q(G), x)=\sum_{i} q_{i}(G) x^{n-i}$. Then,

(i) $q_{0}(G)=1$

(ii) $q_{1}(G)=-2 m$

(iii) $q_{2}(G)=2 m^{2}+m-(1 / 2) \sum_{i} d_{i}^{2}$

(iv) $q_{3}(G)=-(1 / 3) \sum_{i} d_{i}^{3}+(m+1) \sum_{i} d_{i}^{2}-(4 / 3) m^{3}-$ $2 m^{2}-2 c_{3}(G)$

(v) $q_{4}(G)=-(1 / 4) \sum_{i} d_{i}^{4}+((2 / 3) m+1) \sum_{i} d_{i}^{3}-(1 / 2)$ $\left(2 m^{2}+5 m+1\right) \sum_{i} d_{i}^{2}+(1 / 8)\left(\sum_{i} d_{i}^{2}\right)^{2}+\sum_{\left(v_{i} v_{j}\right) \in E(G)}$ $d_{i} d_{j}-2 \sum_{i} d_{i} c_{3}\left(G_{v_{i}}\right)+2 c_{4}(G)+4 m c_{3}(G)+(2 / 3)$ $m^{4}+2 m^{3}+(1 / 2) m^{2}+(1 / 2) m$ 


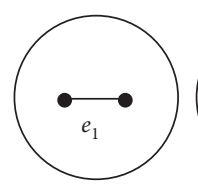

$G_{10}$

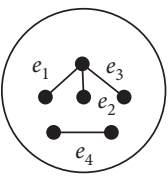

$G_{41}$

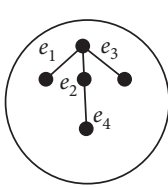

$G_{410}$
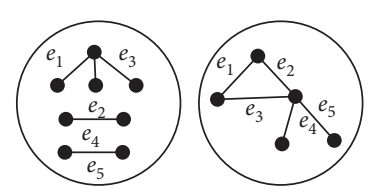

$G_{58}$

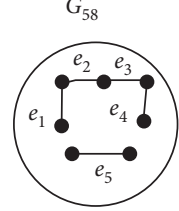

$G_{517}$

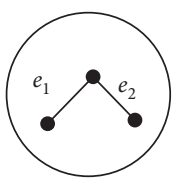

$G_{20}$

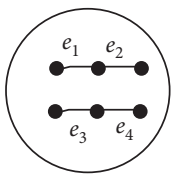

$G_{42}$

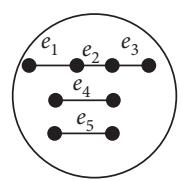

$G_{50}$

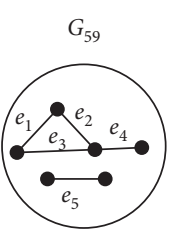

$G_{518}$

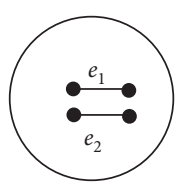

$G_{21}$

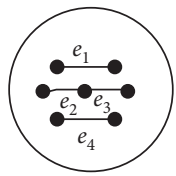

$G_{43}$

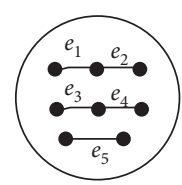

$G_{51}$
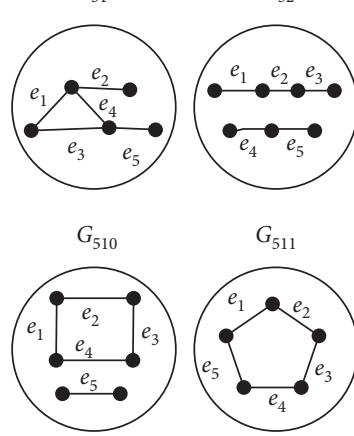

$G_{519}$

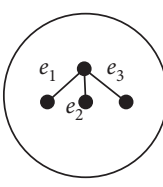

$G_{30}$
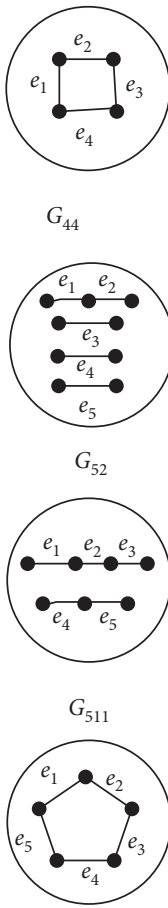

$G_{520}$

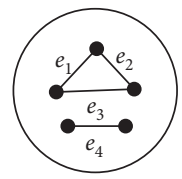

$G_{45}$

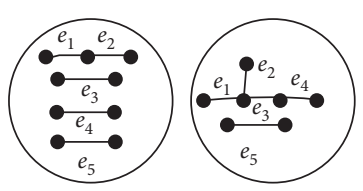

$G_{53}$
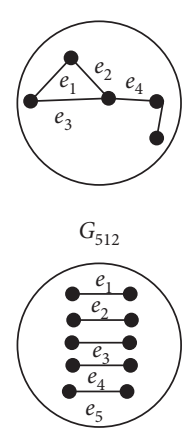

$G_{521}$

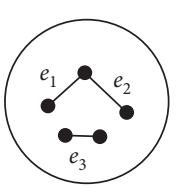

$G_{31}$

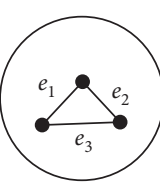

$G_{32}$

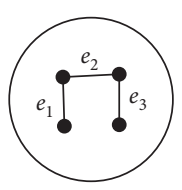

$G_{33}$

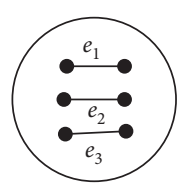

$G_{34}$
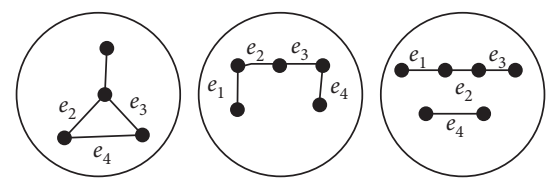

$G_{48}$
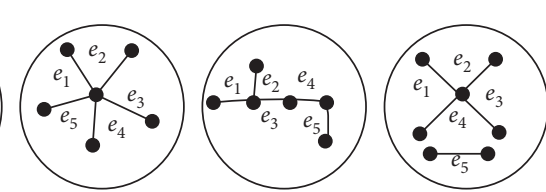

$G_{56}$
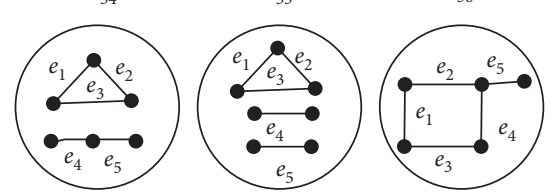

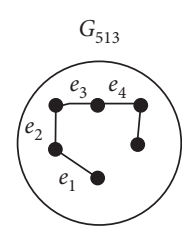

$G_{522}$

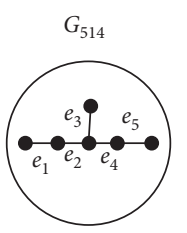

$G_{523}$

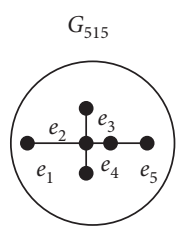

$G_{524}$
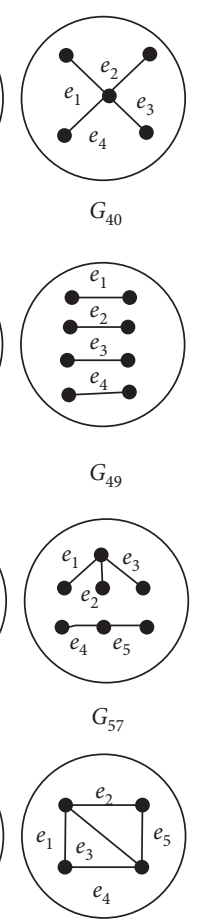

$G_{40}$

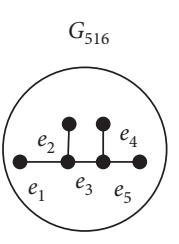

$G_{525}$

FIgure 1: The graphs obtained from $K_{n}$ by deleting five or fewer edges drawn as lines in a disk.

TABLE 1: The numbers of triangles of some graphs in $\mathscr{G}_{n}$

\begin{tabular}{ll}
\hline Graph & $c_{3}(G)$ \\
\hline$G_{30}$ & $\left(\begin{array}{l}n \\
3\end{array}\right)-3 n+9$ \\
$G_{32}, G_{33}$ & $\left(\begin{array}{l}n \\
3\end{array}\right)-3 n+8$ \\
$G_{41}, G_{47}$ & $\left(\begin{array}{l}n \\
3\end{array}\right)-4 n+11$ \\
$G_{44}, G_{46}, G_{410}$ & $\left(\begin{array}{c}n \\
3\end{array}\right)-4 n+12$ \\
$G_{50}, G_{51}, G_{514}$ & $\left(\begin{array}{l}n \\
3\end{array}\right)-5 n+12$ \\
$G_{52}$ & $\left(\begin{array}{c}n \\
3\end{array}\right)-5 n+11$ \\
$G_{54}$ & $\left(\begin{array}{c}n \\
3\end{array}\right)-5 n+20$ \\
$G_{53}, G_{57}, G_{518}, G_{519}, G_{522}$ & $\left(\begin{array}{c}n \\
3\end{array}\right)-5 n+14$ \\
$G_{31}$ & $\left(\begin{array}{c}n \\
3\end{array}\right)-3 n+7$ \\
$G_{40}$ & $\left(\begin{array}{c}n \\
3\end{array}\right)-4 n+14$ \\
\hline
\end{tabular}

TABle 1: Continued.

\begin{tabular}{lc}
\hline Graph & $c_{3}(G)$ \\
\hline$G_{43}$ & $\left(\begin{array}{l}n \\
3\end{array}\right)-4 n+9$ \\
$G_{42}, G_{45}, G_{48}$ & $\left(\begin{array}{l}n \\
3\end{array}\right)-4 n+10$ \\
$G_{59}, G_{524}$ & $\left(\begin{array}{l}n \\
3\end{array}\right)-5 n+17$ \\
$G_{55}, G_{512}, G_{520}, G_{523}$ & $\left(\begin{array}{l}n \\
3\end{array}\right)-5 n+15$ \\
$G_{58}, G_{511}, G_{513}, G_{517}$ & $\left(\begin{array}{l}n \\
3\end{array}\right)-5 n+13$ \\
$G_{56}, G_{510}, G_{515}, G_{516}, G_{525}$ & $\left(\begin{array}{l}n \\
3\end{array}\right)-5 n+16$ \\
\hline
\end{tabular}

By Lemmas 4 and 5, we have the following.

Corollary 1. Let $G$ be a graph with $n$ vertices and $m$ edges, and let $\left(d_{1}, d_{2}, \ldots, d_{n}\right)$ be the degree sequence of $G$. Suppose that $\pi(L(G), x)=\sum_{i} p_{i}(G) x^{n-i}$ and $\pi(Q(G), x)=\sum_{i} q_{i}$ (G) $x^{n-i}$. Then, 
TABLe 2: The numbers of quadrangles of some graphs in $\mathscr{G}_{n}$.

\begin{tabular}{|c|c|}
\hline Graph & $c_{4}(G)$ \\
\hline$G_{32}$ & $3\left(\begin{array}{c}n \\
4\end{array}\right)-3 n^{2}+18 n-27$ \\
\hline$G_{42}$ & $-4 n^{2}+22 n-22$ \\
\hline$G_{50}$ & $-5 n^{2}+27 n-21$ \\
\hline$G_{53}$ & $-5 n^{2}+29 n-32$ \\
\hline$G_{55}$ & $-5 n^{2}+30 n-38$ \\
\hline$G_{511}$ & $-5 n^{2}+28 n-26$ \\
\hline$G_{58}, G_{514}$ & $-5 n^{2}+28 n-25$ \\
\hline$G_{512}, G_{525}$ & $-5 n^{2}+31 n-44$ \\
\hline$G_{520}$ & $-5 n^{2}+30 n-40$ \\
\hline$G_{41}, G_{45}$ & $-4 n^{2}+23 n-27$ \\
\hline$G_{48}$ & $-4 n^{2}+22 n-23$ \\
\hline$G_{51}$ & $-5 n^{2}+27 n-20$ \\
\hline$G_{57}$ & $-5 n^{2}+29 n-30$ \\
\hline$G_{523}$ & $-5 n^{2}+30 n-39$ \\
\hline$G_{517}$ & $-5 n^{2}+28 n-27$ \\
\hline$G_{519}, G_{522}$ & $3\left(\begin{array}{c}n \\
4\end{array}\right)-5 n^{2}+29 n-33$ \\
\hline$G_{518}$ & $3\left(\begin{array}{l}n \\
4\end{array}\right)-5 n^{2}+30 n-37$ \\
\hline
\end{tabular}

(i) $p_{3}(G)-q_{3}(G)=4 c_{3}(G)$

(ii) $p_{4}(G)-q_{4}(G)=4 \sum_{i} d_{i} c_{3}\left(G_{v_{i}}\right)-8 m c_{3}(G)$

For convenience, we calculate the value $\sum_{i} d_{i} c_{3}\left(G_{v_{i}}\right)$ of some graphs in $\mathscr{G}_{n}$, see Table 3.

Lemma 6 (see [19]). The following can be deduced from the (signless) Laplacian permanental polynomial of a graph $G$ :
TABLE 3: The value of $\sum_{i} d_{i} c_{3}\left(G_{v_{i}}\right)$ of some graphs in $\mathscr{G}_{n}$.

\begin{tabular}{ll}
\hline Graph & $\sum_{i} d_{i} c_{3}\left(G_{v_{i}}\right)$ \\
\hline$G_{30}$ & $(1 / 2) n^{4}-2 n^{3}-(19 / 2) n^{2}+56 n-66$ \\
$G_{41}$ & $(1 / 2) n^{4}-2 n^{3}-(27 / 2) n^{2}+70 n-66$ \\
$G_{42}$ & $(1 / 2) n^{4}-2 n^{3}-(27 / 2) n^{2}+65 n-50$ \\
$G_{50}$ & $(1 / 2) n^{4}-2 n^{3}-(35 / 2) n^{2}+79 n-48$ \\
$G_{53}$ & $(1 / 2) n^{4}-2 n^{3}-(35 / 2) n^{2}+89 n-79$ \\
$G_{58}$ & $(1 / 2) n^{4}-2 n^{3}-(35 / 2) n^{2}+84 n-62$ \\
$G_{519}$ & $(1 / 2) n^{4}-2 n^{3}-(35 / 2) n^{2}+89 n-80$ \\
$G_{511}$ & $(1 / 2) n^{4}-2 n^{3}-(35 / 2) n^{2}+84 n-61$ \\
$G_{55}$ & $(1 / 2) n^{4}-2 n^{3}-(35 / 2) n^{2}+94 n-94$ \\
$G_{512}$ & $(1 / 2) n^{4}-2 n^{3}-(35 / 2) n^{2}+96 n-105$ \\
$G_{518}$ & $(1 / 2) n^{4}-2 n^{3}-(35 / 2) n^{2}+91 n-88$ \\
$G_{513}$ & $(1 / 2) n^{4}-2 n^{3}-(35 / 2) n^{2}+86 n-69$ \\
$G_{32}$ & $(1 / 2) n^{4}-2 n^{3}-(19 / 2) n^{2}+53 n-60$ \\
$G_{45}$ & $(1 / 2) n^{4}-2 n^{3}-(27 / 2) n^{2}+67 n-60$ \\
$G_{48}$ & $(1 / 2) n^{4}-2 n^{3}-(27 / 2) n^{2}+65 n-52$ \\
$G_{51}$ & $(1 / 2) n^{4}-2 n^{3}-(35 / 2) n^{2}+79 n-46$ \\
$G_{57}$ & $(1 / 2) n^{4}-2 n^{3}-(35 / 2) n^{2}+89 n-75$ \\
$G_{514}$ & $(1 / 2) n^{4}-2 n^{3}-(35 / 2) n^{2}+81 n-56$ \\
$G_{522}$ & $(1 / 2) n^{4}-2 n^{3}-(35 / 2) n^{2}+89 n-78$ \\
$G_{517}$ & $(1 / 2) n^{4}-2 n^{3}-(35 / 2) n^{2}+84 n-63$ \\
$G_{523}$ & $(1 / 2) n^{4}-2 n^{3}-(35 / 2) n^{2}+94 n-96$ \\
$G_{525}$ & $(1 / 2) n^{4}-2 n^{3}-(35 / 2) n^{2}+99 n-112$ \\
$G_{520}$ & $(1 / 2) n^{4}-2 n^{3}-(35 / 2) n^{2}+94 n-95$ \\
\hline
\end{tabular}

(i) The number of vertices

(ii) The number of edges

(iii) The sum of the squares of degree of vertices

We recorded the results of the sum of squares of degrees of some graphs in advance, see Table 4.

\section{The Proof of Theorem 1}

We give some lemmas to prove Theorem 1 before. First, by Lemma 6 and Table 4, we obtain a result as follows.

Lemma 8. Graphs $K_{n}, G_{10}, G_{20}, G_{21}, G_{31}, G_{33}, G_{34}, G_{40}, G_{43}$, $G_{46}, G_{49}, G_{52}, G_{54}$, and $G_{521}$ are determined by the (signless) Laplacian permanental polynomial, respectively.

Lemma 9. Graphs $G_{30}$ and $G_{32}$ are determined by the (signless) Laplacian permanental polynomial.

Proof. By Table 4, we know that $\sum_{i=1}^{n} d_{i}^{2}\left(G_{30}\right)=\sum_{i=1}^{n}$ $d_{i}^{2}\left(G_{32}\right)=n^{3}-2 n^{2}-11 n+24$.

By Lemma 4 (iv) and Table 1, we have

$$
p_{3}\left(G_{30}\right)-p_{3}\left(G_{32}\right)=-\frac{1}{3}\left(\sum_{i} d_{i}^{3}\left(G_{30}\right)-\sum_{i} d_{i}^{3}\left(G_{32}\right)\right)+2\left(c_{3}\left(G_{30}\right)-c_{3}\left(G_{32}\right)\right)=4
$$


TABle 4: The sum of squares of degrees of some graphs in $\mathscr{G}_{n}$.

\begin{tabular}{lc}
\hline Graph & $\sum_{1 \leq i \leq n} d_{i}^{2}$ \\
\hline$G_{20}$ & $n^{3}-2 n^{2}-7 n+14$ \\
$G_{30}, G_{32}$ & $n^{3}-2 n^{2}-11 n+24$ \\
$G_{33}$ & $n^{3}-2 n^{2}-11 n+22$ \\
$G_{40}$ & $n^{3}-2 n^{2}-15 n+36$ \\
$G_{42}, G_{48}$ & $n^{3}-2 n^{2}-15 n+28$ \\
$G_{44}, G_{410}$ & $n^{3}-2 n^{2}-15 n+32$ \\
$G_{49}$ & $n^{3}-2 n^{2}-15 n+24$ \\
$G_{52}$ & $n^{3}-2 n^{2}-19 n+32$ \\
$G_{54}$ & $n^{3}-2 n^{2}-19 n+50$ \\
$G_{56}, G_{512}, G_{515}, G_{525}$ & $n^{3}-2 n^{2}-19 n+42$ \\
$G_{58}, G_{511}, G_{514}, G_{517}$ & $n^{3}-2 n^{2}-19 n+36$ \\
$G_{510}, G_{524}$ & $n^{3}-2 n^{2}-19 n+44$ \\
$G_{21}$ & $n^{3}-2 n^{2}-7 n+12$ \\
$G_{31}$ & $n^{3}-2 n^{2}-11 n+20$ \\
$G_{34}$ & $n^{3}-2 n^{2}-11 n+18$ \\
$G_{41}, G_{45}, G_{47}$ & $n^{3}-2 n^{2}-15 n+30$ \\
$G_{43}$ & $n^{3}-2 n^{2}-15 n+26$ \\
$G_{46}$ & $n^{3}-2 n^{2}-15 n+34$ \\
$G_{50}, G_{51}$ & $n^{3}-2 n^{2}-19 n+34$ \\
$G_{53}, G_{57}, G_{513}, G_{519}, G_{522}$ & $n^{3}-2 n^{2}-19 n+38$ \\
$G_{55}, G_{518}, G_{520}, G_{523}$ & $n^{3}-2 n^{2}-19 n+40$ \\
$G_{521}$ & $n^{3}-2 n^{2}-19 n+30$ \\
$G_{59}, G_{516}$ & $n^{3}-2 n^{2}-19 n+46$ \\
\hline &
\end{tabular}

Furthermore, by Lemma 5 (v) and Tables $1-3$, we have

$$
\begin{aligned}
q_{4}\left(G_{32}\right)-q_{4}\left(G_{30}\right) \\
=-\frac{1}{4}\left(\sum_{i} d_{i}^{4}\left(G_{32}\right)-\sum_{i} d_{i}^{4}\left(G_{30}\right)\right)+\left(\frac{2}{3} m+1\right) \\
+\left(\sum_{i} d_{i}^{3}\left(G_{32}\right)-\sum_{i} d_{i}^{3}\left(G_{30}\right)\right) \\
+\left(\sum_{\left(v_{i} v_{j}\right) \in E\left(G_{32}\right)} d_{i} d_{j}-\sum_{\left(v_{i} v_{j}\right) \in E\left(G_{30}\right)} d_{i} d_{j}\right) \\
+2\left(\sum_{i} d_{i} c_{3}\left(G_{v_{32}}\right)+\sum_{i} d_{i} c_{3}\left(G_{v_{30}}\right)\right) \\
+2\left(c_{4}\left(G_{32}\right)-c_{4}\left(G_{30}\right)\right)+4 m\left(c_{3}\left(G_{32}\right)-c_{3}\left(G_{30}\right)\right)=-6 .
\end{aligned}
$$

These imply that $G_{30}$ and $G_{32}$ are not (signless) Laplacian copermanental.

Lemma 10. The following statements hold:

(i) Graphs $G_{41}, G_{45}$, and $G_{47}$ are not pairwise (signless) Laplacian copermanental (ii) Graphs $G_{42}$ and $G_{48}$ are not (signless) Laplacian copermanental

(iii) Graphs $G_{44}$ and $G_{410}$ are not (signless) Laplacian copermanental

Proof

(i) By Lemma 4 (iv) and Table 1, we get that $p_{3}\left(G_{41}\right)-$ $p_{3}\left(G_{45}\right)=4, p_{3}\left(G_{41}\right)-p_{3}\left(G_{47}\right)=2$ and $p_{3}\left(G_{45}\right)-$ $p_{3}\left(G_{47}\right)=2$. Furthermore, by Corollary 1 (i), Table 1 , and the equations above, we obtain that $q_{3}\left(G_{41}\right)-q_{3}\left(G_{45}\right)=4-4\left(c_{3}\left(G_{41}\right)-c_{3}\left(G_{45}\right)\right)=0$, $q_{3}\left(G_{41}\right)-q_{3}\left(G_{47}\right)=2-4\left(c_{3}\left(G_{41}\right)-c_{3}\left(G_{47}\right)\right)=2$, and $q_{3}\left(G_{45}\right)-q_{3}\left(G_{47}\right)=2-4\left(c_{3}\left(G_{45}\right)-c_{3}\left(G_{47}\right)\right)$ $=6$. Furthermore, by Lemma $5(\mathrm{v})$ and Tables $1-3$, we have $q_{4}\left(G_{41}\right)-q_{4}\left(G_{45}\right)=-2$. These mean that graphs $G_{41}, G_{45}$, and $G_{47}$ are not pairwise (signless) Laplacian copermanental.

(ii) From Table 4, we obtain that $\sum_{i=1}^{n} d_{i}^{2}\left(G_{42}\right)$ $=\sum_{i=1}^{n} d_{i}^{2}\left(G_{48}\right)=n^{3}-2 n^{2}-15 n+28$. By Lemma 4 (v) and Tables $1-3$, we have $p_{4}\left(G_{48}\right)-p_{4}\left(G_{42}\right)=-7$. By Corollary 1 (ii), $p_{4}\left(G_{48}\right)-p_{4}\left(G_{42}\right)=-7$, Tables 1 and 3, and the equation above, we obtain that $q_{4}\left(G_{48}\right)-q_{4}\left(G_{42}\right)=-7-4 \sum_{i} d_{i} c_{3}\left(G_{v_{48}}\right)-\sum_{i} d_{i} c_{3}$ $\left(G_{v_{42}}\right)+8 m\left(c_{3}\left(G_{48}\right)-c_{3}\left(G_{42}\right)\right)=1$. This implies that $G_{42}$ and $G_{48}$ are not (signless) Laplacian copermanental.

(iii) Similarly, by Lemma 4 (iv) and Table 1, we have $p_{3}\left(G_{44}\right)-p_{3}\left(G_{410}\right)=-2$. By Corollary $1(\mathrm{i})$, Table 1 and the equation above, we obtain $q_{3}\left(G_{44}\right)-$ $q_{3}\left(G_{410}\right)=-2-4\left(c_{3}\left(G_{44}\right)-c_{3}\left(G_{410}\right)\right)=-2$. These indicate that $G_{44}$ and $G_{410}$ are not (signless) Laplacian copermanental.

Lemma 11. The following statements hold:

(i) Graphs $G_{50}$ and $G_{51}$ are not (signless) Laplacian copermanental

(ii) Graphs $G_{53}, G_{57}, G_{513}, G_{519}$, and $G_{522}$ are not pairwise (signless) Laplacian copermanental

(iii) Graphs $G_{58}, G_{511}, G_{514}$, and $G_{517}$ are not pairwise (signless) Laplacian copermanental

(iv) Graphs $G_{55}, G_{518}, G_{520}$, and $G_{523}$ are not pairwise (signless) Laplacian copermanental

(v) Graphs $G_{59}$ and $G_{516}$ are not (signless) Laplacian copermanental

(vi) Graphs $G_{510}$ and $G_{524}$ are not (signless) Laplacian copermanental

(vii) Graphs $G_{56}, G_{512}, G_{515}$, and $G_{525}$ are not pairwise (signless) Laplacian copermanental 
Proof. By Table 4, we have that

$$
\begin{aligned}
& \sum_{i=1}^{n} d_{i}^{2}\left(G_{50}\right)=\sum_{i=1}^{n} d_{i}^{2}\left(G_{51}\right)=n^{3}-2 n^{2}-19 n+34 \\
& \sum_{i=1}^{n} d_{i}^{2}\left(G_{53}\right)=\sum_{i=1}^{n} d_{i}^{2}\left(G_{57}\right)=\sum_{i=1}^{n} d_{i}^{2}\left(G_{513}\right)=\sum_{i=1}^{n} d_{i}^{2}\left(G_{519}\right)=\sum_{i=1}^{n} d_{i}^{2}\left(G_{522}\right)=n^{3}-2 n^{2}-19 n+38 \\
& \sum_{i=1}^{n} d_{i}^{2}\left(G_{55}\right)=\sum_{i=1}^{n} d_{i}^{2}\left(G_{518}\right)=\sum_{i=1}^{n} d_{i}^{2}\left(G_{520}\right)=\sum_{i=1}^{n} d_{i}^{2}\left(G_{523}\right)=n^{3}-2 n^{2}-19 n+40 \\
& \sum_{i=1}^{n} d_{i}^{2}\left(G_{56}\right)=\sum_{i=1}^{n} d_{i}^{2}\left(G_{512}\right)=\sum_{i=1}^{n} d_{i}^{2}\left(G_{515}\right)=\sum_{i=1}^{n} d_{i}^{2}\left(G_{525}\right)=n^{3}-2 n^{2}-19 n+42 \\
& \sum_{i=1}^{n} d_{i}^{2}\left(G_{58}\right)=\sum_{i=1}^{n} d_{i}^{2}\left(G_{511}\right)=\sum_{i=1}^{n} d_{i}^{2}\left(G_{514}\right)=\sum_{i=1}^{n} d_{i}^{2}\left(G_{517}\right)=n^{3}-2 n^{2}-19 n+36 \\
& \sum_{i=1}^{n} d_{i}^{2}\left(G_{59}\right)=\sum_{i=1}^{n} d_{i}^{2}\left(G_{516}\right)=n^{3}-2 n^{2}-19 n+46 \\
& \sum_{i=1}^{n} d_{i}^{2}\left(G_{56}\right)=\sum_{i=1}^{n} d_{i}^{2}\left(G_{512}\right)=\sum_{i=1}^{n} d_{i}^{2}\left(G_{515}\right)=\sum_{i=1}^{n} d_{i}^{2}\left(G_{525}\right)=n^{3}-2 n^{2}-19 n+42 \\
& \sum_{i=1}^{n} d_{i}^{2}\left(G_{58}\right)=\sum_{i=1}^{n} d_{i}^{2}\left(G_{511}\right)=\sum_{i=1}^{n} d_{i}^{2}\left(G_{514}\right)=\sum_{i=1}^{n} d_{i}^{2}\left(G_{517}\right)=n^{3}-2 n^{2}-19 n+36 \\
& \sum_{i=1}^{n} d_{i}^{2}\left(G_{59}\right)=\sum_{i=1}^{n} d_{i}^{2}\left(G_{516}\right)=n^{3}-2 n^{2}-19 n+46 \\
& \sum_{i=1}^{n} d_{i}^{2}\left(G_{524}\right)=n^{3}-2 n^{2}-19 n+44
\end{aligned}
$$

(i) By Lemma 4 (v) and Tables 1-3, we have $p_{4}\left(G_{50}\right)-p_{4}\left(G_{51}\right)=-7$. By Corollary 1 (ii), Tables 1 and 3 , and the equation above, we get that $q_{4}\left(G_{50}\right)-q_{4}\left(G_{51}\right)=1$. These mean that $G_{50}$ and $G_{51}$ are not (signless) Laplacian copermanental.

(ii) By Lemma 4 (iv) and (v) and Tables 1-3, we get that $p_{4}\left(G_{53}\right)-p_{4}\left(G_{57}\right)=-14, p_{3}\left(G_{53}\right)-p_{3}\left(G_{513}\right)=4$, $p_{3}\left(G_{53}\right)-p_{3}\left(G_{519}\right)=2, \quad p_{3}\left(G_{53}\right)-p_{3}\left(G_{522}\right)=2$, $p_{3}\left(G_{57}\right)-p_{3}\left(G_{513}\right)=4, \quad p_{3}\left(G_{57}\right)-p_{3}\left(G_{519}\right)=2$, $p_{3}\left(G_{57}\right)-p_{3}\left(G_{522}\right)=2, p_{3}\left(G_{513}\right)-p_{3}\left(G_{519}\right)=-2$, $p_{3}\left(G_{513}\right)-p_{3}\left(G_{522}\right)=-2$, and $p_{4}\left(G_{519}\right)-p_{4}$ $\left(G_{522}\right)=-5$.

By Corollary 1, Tables 1 and 3, and the equations above, we obtain that $p_{4}\left(G_{519}\right)-p_{4}\left(G_{522}\right)=-5$, $q_{4}\left(G_{53}\right)-q_{4}\left(G_{513}\right)=-4, \quad q_{3}\left(G_{53}\right)-q_{3}\left(G_{519}\right)=2$, $q_{3}\left(G_{53}\right)-q_{3}\left(G_{522}\right)=2, \quad q_{4}\left(G_{57}\right)-q_{4}\left(G_{513}\right)=-6$, $q_{3}\left(G_{57}\right)-q_{3}\left(G_{519}\right)=2, \quad q_{3}\left(G_{57}\right)-q_{3}\left(G_{522}\right)=2$, $q_{3}\left(G_{513}\right)-q_{3}\left(G_{519}\right)=2, \quad q_{3}\left(G_{513}\right)-q_{3}\left(G_{522}\right)=2$, and $q_{4}\left(G_{519}\right)-q_{4}\left(G_{522}\right)=3$. These imply that $G_{53}$, $G_{57}, G_{513}, G_{519}$, and $G_{522}$ are not pairwise (signless) Laplacian copermanental.

(iii) Similarly, by Lemma 4 (iv) and (v) and Tables 1-3, we have $p_{3}\left(G_{58}\right)-p_{3}\left(G_{511}\right)=2, p_{3}\left(G_{58}\right)-p_{3}$ $\left(G_{514}\right)=4, p_{3}\left(G_{58}\right)-p_{3}\left(G_{517}\right)=2, p_{3}\left(G_{511}\right)-p_{3}$
$\left(G_{514}\right)=2, p_{3}\left(G_{511}\right)-p_{3}\left(G_{517}\right)=0, p_{4}\left(G_{517}\right)-p_{4}$ $\left(G_{511}\right)=-7$, and $p_{3}\left(G_{514}\right)-p_{3}\left(G_{517}\right)=-2$. Furthermore, by Corollary 1, Tables 1 and 3 , and the equations above, we get that $q_{3}\left(G_{58}\right)-q_{3}\left(G_{511}\right)=$ $2, q_{4}\left(G_{58}\right)-q_{4}\left(G_{514}\right)=-6, q_{3}\left(G_{58}\right)-q_{3}\left(G_{517}\right)=2$, $q_{3}\left(G_{511}\right)-q_{3}\left(G_{514}\right)=-2, q_{3}\left(G_{514}\right)-q_{3}\left(G_{517}\right)=2$, and $q_{4}\left(G_{517}\right)-q_{4}\left(G_{511}\right)=1$. These imply that graphs $G_{58}, G_{511}, G_{514}$, and $G_{517}$ are not pairwise (signless) Laplacian copermanental.

(iv) Similarly, by Lemma 4 (iv) and (v) and Tables 1-3, we have $p_{3}\left(G_{55}\right)-p_{3}\left(G_{518}\right)=2, p_{3}\left(G_{55}\right)-p_{3}$ $\left(G_{520}\right)=2, \quad p_{3}\left(G_{55}\right)-p_{3}\left(G_{523}\right)=0, p_{3}\left(G_{518}\right)-p_{3}$ $\left(G_{520}\right)=0, \quad p_{3}\left(G_{518}\right)-p_{3}\left(G_{523}\right)=-2, p_{3}\left(G_{520}\right)-$ $p_{3}\left(G_{523}\right)=-2, \quad p_{4}\left(G_{55}\right)-p_{4}\left(G_{523}\right)=7, \quad$ and $p_{4}\left(G_{518}\right)-p_{4}\left(G_{520}\right)=-1$. By Corollary 1 , Tables 1 and 3 , and the equations above, we know that $q_{3}\left(G_{55}\right)-q_{3}\left(G_{518}\right)=-2, q_{3}\left(G_{55}\right)-q_{3}\left(G_{520}\right)=2, q_{4}$ $\left(G_{55}\right)-q_{4}\left(G_{523}\right)=-1, q_{3}\left(G_{518}\right)-q_{3}\left(G_{520}\right)=4, q_{3}$ $\left(G_{518}\right)-q_{3}\left(G_{523}\right)=2$, and $q_{3}\left(G_{520}\right)-q_{3}\left(G_{523}\right)$ $=-2$. These mean that graphs $G_{55}, G_{518}, G_{520}$, and $G_{523}$ are not pairwise (signless) Laplacian copermanental.

(v) By Lemma 4 (iv) and Table 1, we get that $p_{3}\left(G_{59}\right)-p_{3}\left(G_{516}\right)=6$. By Corollary 1 (i), Table 1 , 
and the equation above, we have $q_{3}\left(G_{59}\right)-q_{3}\left(G_{516}\right)=2$. These mean that graphs $G_{59}$ and $G_{56}$ are not (signless) Laplacian copermanental.

(vi) By Lemma 4 (iv) and Table 1, we have that $p_{3}\left(G_{510}\right)-p_{3}\left(G_{524}\right)=-6$. By Corollary 1 (i), Table 1 , and the equation above, we obtain that $q_{3}\left(G_{510}\right)-q_{3}\left(G_{524}\right)=-2$. Obviously, graphs $G_{510}$ and $G_{524}$ are not (signless) Laplacian copermanental.

(vii) By Lemma 4 (iv) and Table 1 , we have $p_{3}\left(G_{56}\right)-$ $p_{3}\left(G_{512}\right)=8, p_{3}\left(G_{56}\right)-p_{3}\left(G_{515}\right)=6, p_{3}\left(G_{56}\right)-p_{3}$ $\left(G_{525}\right)=4, \quad p_{3}\left(G_{512}\right)-p_{3}\left(G_{515}\right)=-2, p_{3}\left(G_{512}\right)-$ $p_{3}\left(G_{525}\right)=-4$, and $p_{3}\left(G_{515}\right)-p_{3}\left(G_{525}\right)=-2$. Furthermore, by Corollary 1, Tables $1-3$, and the equations above, we have $q_{3}\left(G_{56}\right)-q_{3}\left(G_{512}\right)=4$, $q_{3}\left(G_{56}\right)-q_{3}\left(G_{515}\right)=6, \quad q_{3}\left(G_{56}\right)-q_{3}\left(G_{525}\right)=4$, $q_{3}\left(G_{512}\right)-q_{3}\left(G_{515}\right)=2, \quad q_{3}\left(G_{512}\right)-q_{3}\left(G_{525}\right)=6$, $q_{3}\left(G_{515}\right)-q_{3}\left(G_{525}\right)=-2$, and $q_{4}\left(G_{512}\right)-q_{4}$ $\left(G_{525}\right)=24$. Obviously, graphs $G_{56}, G_{512}, G_{515}$, and $G_{525}$ are not pairwise (signless) Laplacian copermanental.

Proof of Theorem 1. From Lemmas 6-11, we directly obtain Theorem 1.

\section{Data Availability}

No data were used to support this study.

\section{Conflicts of Interest}

The authors declare that they have no conflicts of interest or personal relationships that could have appeared to influence the work reported in this paper.

\section{Acknowledgments}

This work was supported by the National Natural Science Foundation of China (no. 11761056), by Natural Science Foundation of Qinghai Province (no. 2020-ZJ-920), and the Scientific Research Innovation Team in Qinghai Nationalities University.

\section{References}

[1] L. G. Valiant, "The complexity of computing the permanent," Theoretical Computer Science, vol. 8, no. 2, pp. 189-201, 1979.

[2] R. Merris, K. R. Rebman, and W. Watkins, "Permanental polynomials of graphs," Linear Algebra and Its Applications, vol. 38, pp. 273-288, 1981.

[3] I. Faria, "Permanental roots and the star degree of a graph," Linear Algebra and Its Applications, vol. 64, pp. 255-265, 1985.

[4] P. Botti, R. Merris, and C. Vega, "Laplacian permanents of trees," SIAM Journal on Discrete Mathematics, vol. 5, no. 4, pp. 460-466, 1992.

[5] R. A. Brualdi and J. L. Goldwasser, "Permanent of the Laplacian matrix of trees and bipartite graphs," Discrete Mathematics, vol. 48, no. 1, pp. 1-21, 1984.
[6] G. G. Cash and I. Gutman, "The Laplacian permanental polynomial: formulas and algorithms," MATCH-Communications in Mathematical and in Computer Chemistry, vol. 51, pp. 129-136, 2004.

[7] X. Geng, S. Hu, and S. Li, "Permanental bounds of the Laplacian matrix of trees with given domination number," Graphs and Combinatorics, vol. 31, no. 5, pp. 1423-1436, 2015.

[8] Y. Shi, M. Dehmer, X. Li, and I. Gutman, Graph Polynomials, CRC Press, Boca Raton, FL, USA, 2017.

[9] S. Li and L. Zhang, "Permanental bounds for the signless Laplacian matrix of bipartite graphs and unicyclic graphs," Linear and Multilinear Algebra, vol. 59, no. 2, pp. 145-158, 2011.

[10] S. Li and L. Zhang, "Permanental bounds for the signless Laplacian matrix of a unicyclic graph with diameter d," Graphs and Combinatorics, vol. 28, no. 4, pp. 531-546, 2012.

[11] X. Liu and T. Wu, "Computing the permanental polynomials of graphs," Applied Mathematics and Computation, vol. 304, pp. 103-113, 2017.

[12] X. Liu and T. Wu, "On the normalized Laplacian permanental polynomial of a graph," Bulletin of the Iranian Mathematical Society, vol. 45, no. 5, pp. 1303-1321, 2019.

[13] R. Merris, "The Laplacian permanental polynomial for trees," Czechoslovak Mathematical Journal, vol. 32, no. 3, pp. 397403, 1982.

[14] T. Wu and W. So, "Permanental sums of graphs of extreme sizes," Discrete Mathematics, vol. 344, Article ID 112353, 2021.

[15] D. Cvetković, M. Doob, and H. Sachs, Spectra of Graphs, Academic Press, New York, NY, USA, 1982.

[16] D. Cvetković, P. Rowlinson, and S. Simić, An Introduction to the Theory of Graph Spectra, Cambridge University Press, Cambridge, UK, 2010.

[17] E. R. van Dam and W. H. Haemers, "Which graphs are determined by their spectrum?" Linear Algebra and Its Applications, vol. 373, pp. 241-272, 2003.

[18] E. R. van Dam and W. H. Haemers, "Developments on spectral characterizations of graphs," Discrete Mathematics, vol. 309, no. 3, pp. 576-586, 2009.

[19] S. Liu, "On the (Signless) Laplacian permanental polynomials of graphs," Graphs and Combinatorics, vol. 35, no. 3, pp. 787-803, 2019.

[20] M. Cámara and W. H. Haemers, "Spectral characterizations of almost complete graphs," Discrete Applied Mathematics, vol. 176, pp. 19-23, 2014.

[21] T. Wu and T. Zhou, " $A_{\alpha}$-Spectral characterizations of some joins," Journal of Mathematics, vol. 2020, Article ID 8294312, 8 pages, 2020.

[22] H. Zhang, T. Wu, and H.-J. Lai, "Per-spectral characterizations of some edge-deleted subgraphs of a complete graph," Linear and Multilinear Algebra, vol. 63, no. 2, pp. 397-410, 2015. 